\title{
Caracterização microestrutural de materiais cerâmicos utilizando o programa de processamento digital de imagens Image $\mathbf{J}$
}

\section{(Microstructural characterization of ceramic materials using the image digital processing software Image $J$ )}

\author{
R. F. Marcomini, D. M. P. F de Souza \\ Programa de Pós-graduação em Ciência e Engenharia de Materiais, Universidade Federal de S. Carlos, Rod. \\ Washington Luís km 255, S. Carlos, SP
}

\begin{abstract}
Resumo
Neste trabalho foram quantificados o tamanho de grão e a porosidade de uma amostra de zircônia estabilizada com ítria (ZEI) sinterizada a $1500{ }^{\circ} \mathrm{C} / 2 \mathrm{~h}$ e a fração volumétrica das fases $\mathrm{ZEI}, \mathrm{NiO}$ e poros de um compósito de ZEI/NiO sinterizado a $1500{ }^{\circ} \mathrm{C} / 2$ h. Utilizando o programa de análise de imagens ImageJ foi possível tratar as imagens removendo ruídos e evidenciando as regiões de interesse de análise. Após as imagens serem analisadas, foi feito o tratamento estatístico dos dados como sugerido nas normas ASTM E 562-02 e ASTM E 1382-97. As frações volumétricas obtidas desta maneira foram $50 \pm 7 \%$ de NiO, $32 \pm 2 \%$ ZEI e 17 $\pm 7 \%$ poros. O tamanho médio de grãos da ZEI foi $4,6 \pm 0,2 \mu \mathrm{m}$ e sua porosidade $1,5 \pm 0,2 \%$. Os resultados de porosidade foram comparados com os valores obtidos utilizando o método baseado no princípio de Arquimedes. A diferença entre os valores permitiu por este método quantificar somente a porosidade aberta, enquanto a análise de imagens quantifica também os poros fechados.

Palavras-chave: microscopia quantitativa, processamento de análises digitais, análise microestrutural.
\end{abstract}

Abstract

In this work grain size and porosity of Yttria Stabilized Zirconia (YSZ) sintered at $1500^{\circ} \mathrm{C} / 2 \mathrm{~h}$ and the volume fraction of the phases $\mathrm{YSZ}, \mathrm{NiO}$ and pores in a YSZ/ NiO composite sintered at $1500^{\circ} \mathrm{C} / 2 \mathrm{~h}$ were quantified. Using the image analysis software ImageJ it was possible to treat images removing noise and showing the regions of interest in the analysis. After analyzing the images a statistical treatment of the data as suggested in the standards ASTM E 562-02 and ASTM E 1382-97 was carried out. The volume fractions obtained were $50 \pm 7 \% \mathrm{NiO}, 32 \pm 2 \% \mathrm{YSZ}$ and $17 \pm 7 \%$ pores. The average YSZ grain size was $4.6 \pm 0.2 \mu \mathrm{m}$ and the porosity $1.5 \pm 0.2 \%$. The porosity results were compared with values obtained using a method based on the Arquimedes principle; the difference between the values was that this method quantifies only open porosity, while image analysis also quantifies closed pores.

Keywords: quantitative microscopy, digital image processing, microstructural analysis.

\section{INTRODUÇÃO}

O desenvolvimento sistemático de novos materiais depende fortemente de sua caracterização em diversos níveis de resolução. Estrutura, microestrutura e geometria de defeitos, assim como composição química e distribuição espacial são parâmetros importantes para se determinar o comportamento de materiais em aplicações específicas [1].

A determinação manual do tamanho de grãos e fração volumétrica de fases pode ser uma tarefa laboriosa, geralmente sujeita a erros do operador. Como alternativa para minimizar tais erros e diminuir o tempo de análise o uso de programas computacionais de análise de imagem têm sido cada vez mais comuns. Dentre os diversos programas disponíveis no mercado, com custos variáveis, o ImageJ tem se destacado.

ImageJ é um programa de domínio público desenvolvido pelo National Institute of Health, NIH, Estados Unidos. Por ser de domínio público possui seu código aberto possibilitando que a comunidade de usuários desenvolva complementos e modificações de acordo com suas necessidades [2].

A análise de imagem automatizada consiste em tratar uma imagem digital evidenciando as regiões de interesse de maneira que o programa possa realizar a análise. $\mathrm{O}$ tratamento de imagem se dá por operações de matrizes que alteram o valor de seus pixeis. Pixeis são elementos da imagem e possuem posição e valor de brilho definidos, formando uma matriz correspondente à imagem. Existem diversas operações já descritas na literatura e a aplicação consecutiva de diferentes operações, ou filtros, podem levar ao mesmo resultado. Desta maneira, a experiência do operador e o conhecimento básico dos filtros são importantes para se desenvolver um algoritmo para evidenciar as regiões de interesse da imagem [3]. O resultado do tratamento deve ser uma imagem contendo as características que se desejam analisar, no caso deste trabalho serão evidenciados os contornos de grãos e as fases presentes. A partir da imagem tratada e após a calibração do software podem-se realizar as medidas. O resultado da efetividade do uso de certo filtro depende da análise subjetiva do operador, 
no entanto os resultados finais apresentam menores desvios que os resultados obtidos por medidas manuais [4]

Existe uma gama de aplicações na análise digital de imagens, sendo já bastante utilizada no controle de qualidade na produção de aços, determinando-se tanto o tamanho médio de grãos quanto a quantidade de inclusões presentes. Em sistemas cerâmicos informações como tamanho de grão, porosidade, quantidade e disposição de fases podem ser obtidas para se determinar, por exemplo, a temperatura ideal de sinterização, presença de fases deletérias a propriedades de interesse, defeitos inseridos durante o processamento, entre outros, quantificando as correlações entre microestrutura, processamento e propriedades [5-11].

No presente trabalho foram determinadas as frações volumétricas de poros, das fases $\mathrm{NiO}$ e zircônia estabilizada com ítria (ZEI) em um compósito NiO/ZEI e também a porosidade e o tamanho de grãos de uma peça de ZEI. As análises foram realizadas com o programa ImageJ, com imagens obtidas por microscopia eletrônica de varredura.

\section{MATERIAIS E MÉTODOS}

Obtenção do compósito ZEI/NiO para determinação da fração volumétrica das fases

O compósito poroso $44 \% \mathrm{ZEI}-66 \% \mathrm{NiO}$ (\% em peso) foi preparado a partir da mistura aquosa dos componentes Tosoh (TZ-8Y) e Vetec, respectivamente. Os óxidos foram misturados em água destilada e o $\mathrm{pH}$ ajustado até o valor 3 , utilizando solução de $10 \%$ em volume de $\mathrm{HCl}$, medido em pHmetro Micronal B374. Neste valor de pH obtém-se a melhor condição de defloculação determinada previamente por ensaio de medida de viscosidade da suspensão para vários valores do $\mathrm{pH}$. A medida de viscosidade foi realizada em viscosímetro Haake VT-550 em condição cone-placa, sensor PK-100. Após $5 \mathrm{~h}$ de homogeneização, foi adicionado à suspensão amido de milho comercial, tamanho médio de partículas $10 \mu \mathrm{m}$, para atuar como formador de poros. $\mathrm{O} \mathrm{pH}$ foi novamente ajustado em 3 e a suspensão homogeneizada por mais $1 \mathrm{~h}$. A suspensão foi seca ao ar e em seguida granulada em peneira de nylon 80 mesh $(178 \mu \mathrm{m})$. Após a granulação, o pó foi prensado uniaxialmente a $40 \mathrm{MPa}$ na forma de pastilhas de $13 \mathrm{~mm}$ de diâmetro e $1 \mathrm{~mm}$ de espessura e em seguida prensadas isostaticamente a $200 \mathrm{MPa}$ durante 1 min para eliminar os gradientes de densidade. As pastilhas foram sinterizadas com taxa de aquecimento de $150{ }^{\circ} \mathrm{C} / \mathrm{h}$ até $450{ }^{\circ} \mathrm{C}$, mantidas nesta temperatura durante $2 \mathrm{~h}$ para a eliminação do amido de milho e, em seguida, foram aquecidas com taxa de $800^{\circ} \mathrm{C} / \mathrm{h}$ até 1500 ${ }^{\circ} \mathrm{C}$, com patamar de $2 \mathrm{~h}$ visando a sinterização da amostra.

As pastilhas sinterizadas foram fraturadas e embutidas em resina de poliéster insaturado, dissolvida com cerca de $20 \%$ em volume de estireno para reduzir sua viscosidade. O embutimento foi feito sob vácuo para que a resina penetrasse nos poros da amostra, mantendo assim sua integridade estrutural [5]. Após o polimento, as amostras foram analisadas em microscópio eletrônico de varredura TMPPhilips. As imagens foram tratadas para evidenciar as fases e analisadas utilizando o aplicativo ImageJ e a norma ASTM E 562-02 [12]. Foi utilizado o seguinte algoritmo: filtro Sigma Filter Plus; normalização e equalização do histograma; limiarização da imagem separando as fases de interesse. Este procedimento foi realizado em 5 imagens, de onde foram extraídas as informações de fração volumétrica de poros, ZEI e NiO. Como referência para o valor da porosidade fez-se a medida pelo princípio de Arquimedes. Importante destacar que a porosidade medida por este método é devida aos poros abertos enquanto que a obtida pela análise de imagem é a porosidade total, isto é, poros abertos e fechados.

Obtenção de amostra de ZEI para quantificação do tamanho de grão e porosidade

Foi preparada uma suspensão contendo $20 \%$ em volume de ZEI (Tosoh TZ-8Y) e 1\% em massa em relação à quantidade de óxidos de poli-vinil butiral (Solutia PVB Butvar B98) solubilizado em álcool isopropílico. Esta suspensão foi homogeneizada durante $6 \mathrm{~h}$ em moinho vibratório, seca e granulada utilizando uma peneira de 80 mesh. Pastilhas com $13 \mathrm{~mm}$ de diâmetro foram prensadas isostaticamente a 200 MPa e em seguida sinterizadas a $1500{ }^{\circ} \mathrm{C} / 2 \mathrm{~h}$, fraturadas e polidas com pasta de diamante até $1 \mu \mathrm{m}$. As pastilhas polidas foram submetidas a ataque térmico a $1400^{\circ} \mathrm{C} / 30 \mathrm{~min}$ visando revelar os contornos de grão. As amostras foram analisadas em microscópio eletrônico de varredura e as imagens foram tratadas para evidenciar os contornos de grãos. A análise foi feita utilizando o programa ImageJ utilizando o seguinte algoritmo: filtro subtrair fundo; filtro Find Edges; filtro Make Binary. Após o uso deste procedimento foram aplicadas as operações Dilate e Erode para fechar os contornos de grãos permitindo que o programa interprete cada grão como um objeto único. Foram analisadas 7 imagens, atingindo a contagem de mais de 600 grãos. Os dados foram tratados seguindo a norma ASTM E 1382-97 [13].

Para a quantificação da porosidade foi necessária somente a limiarização das imagens. Foram analisadas 5 imagens e o tratamento dos dados foi feito conforme proposto na referência [12]. Os dados foram comparados com os valores obtidos utilizando a medida realizada pelo princípio de Arquimedes.

\section{RESULTADOS E DISCUSSÃO}

\section{Quantificação das fases por análise de imagens}

A Fig. 1 apresenta uma micrografia obtida por microscopia eletrônica de varredura do compósito poroso $\mathrm{ZEI} / \mathrm{NiO}$. As regiões mais escuras representam os poros, as cinza escuro representam a rede percolada de $\mathrm{NiO}$ e a cinza claro representa a rede percolada de ZEI. A imagem apresenta ruídos, isto é, pixeis apresentando intensidade de brilho diferente dos pixeis vizinhos; além disso, a imagem também possui baixo contraste, isto é, as cores das três diferentes fases possuem intensidades próximas, dificultando a distinção entre elas.

A primeira etapa da análise da micrografia utilizando o programa ImageJ foi a aplicação do filtro Sigma Filter Plus 
[14]. Este filtro suaviza a imagem tomando o valor médio dos pixeis vizinhos, incluindo somente os que contém um valor que não desvie do valor do pixel de referência dentro de uma faixa definida pelo usuário, eliminando assim os ruídos da imagem, preservando interfaces [14]. A Fig. 2 mostra a mesma imagem da Fig. 1, porém tratada com o filtro Sigma Filter Plus. Os ruídos foram reduzidos, mas a diferença de cores entre as fases ainda é pequena.

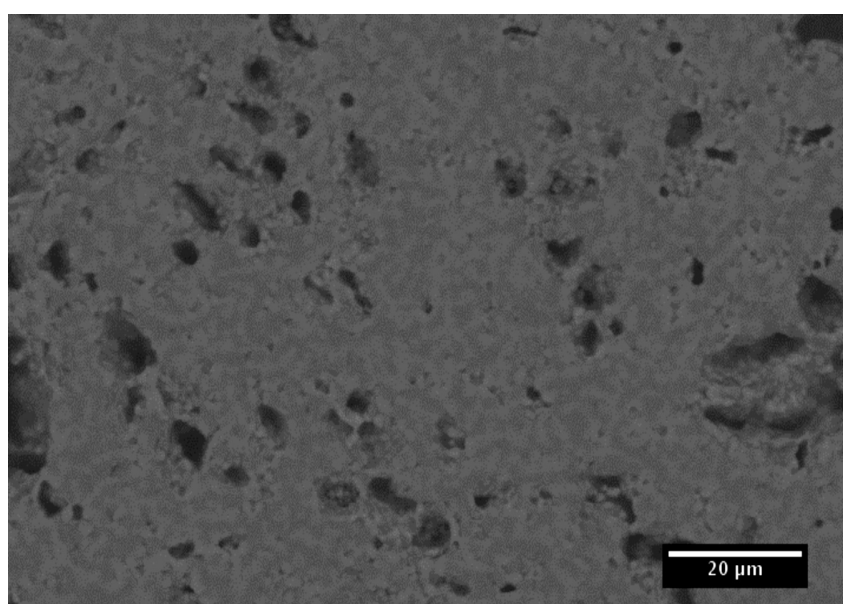

Figura 1: Micrografia obtida em microscópio eletrônico de varredura de um compósito $\mathrm{NiO}$ /zircônia estabilizada com ítrio sinterizado a $1500{ }^{\circ} \mathrm{C} / 2 \mathrm{~h}$.

[Figure 1: SEM micrograph of a NiO/Yttria-stabilized Zirconia composite sintered at $1500^{\circ} \mathrm{C} / 2 \mathrm{~h}$.]

Para acentuar a diferença de cores entre as três fases foi aplicada a ferramenta "Enhance Contrast" (Melhorar Contraste). Contraste é a diferença de valores entre o pixel mais claro e o mais escuro. A normalização do contraste consiste em utilizar toda a faixa de valores possíveis para os pixeis da imagem, por exemplo, em imagens de 8 bits os pixeis mais escuros receberão valor 0 e os mais claros 255 enquanto o restante recebe valores intermediários [15]. A Fig. 3A mostra a imagem e seu histograma de cores não normalizado enquanto a Fig. 3B apresenta a imagem com o histograma normalizado contendo três regiões distintas: uma região mais escura representando os poros, uma região

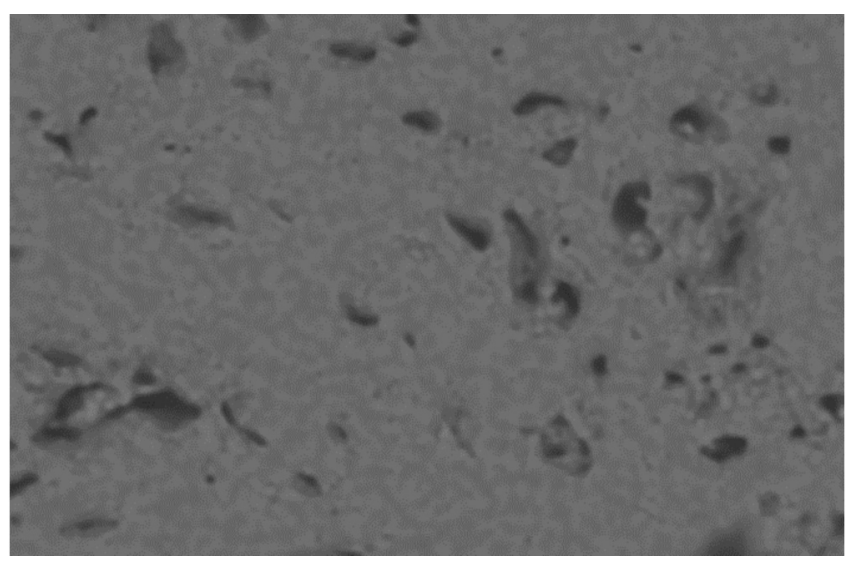

Figura 2: Figura 1 após a aplicação do Filtro Sigma (Sigma Filter). [Figure 2: Same as Fig. 1 after applying Sigma Filter.] de brilho intermediário representando a rede de $\mathrm{NiO}$ e uma região clara que representa a rede de ZEI.

Utilizando a ferramenta "Threshold" (Limiarização),
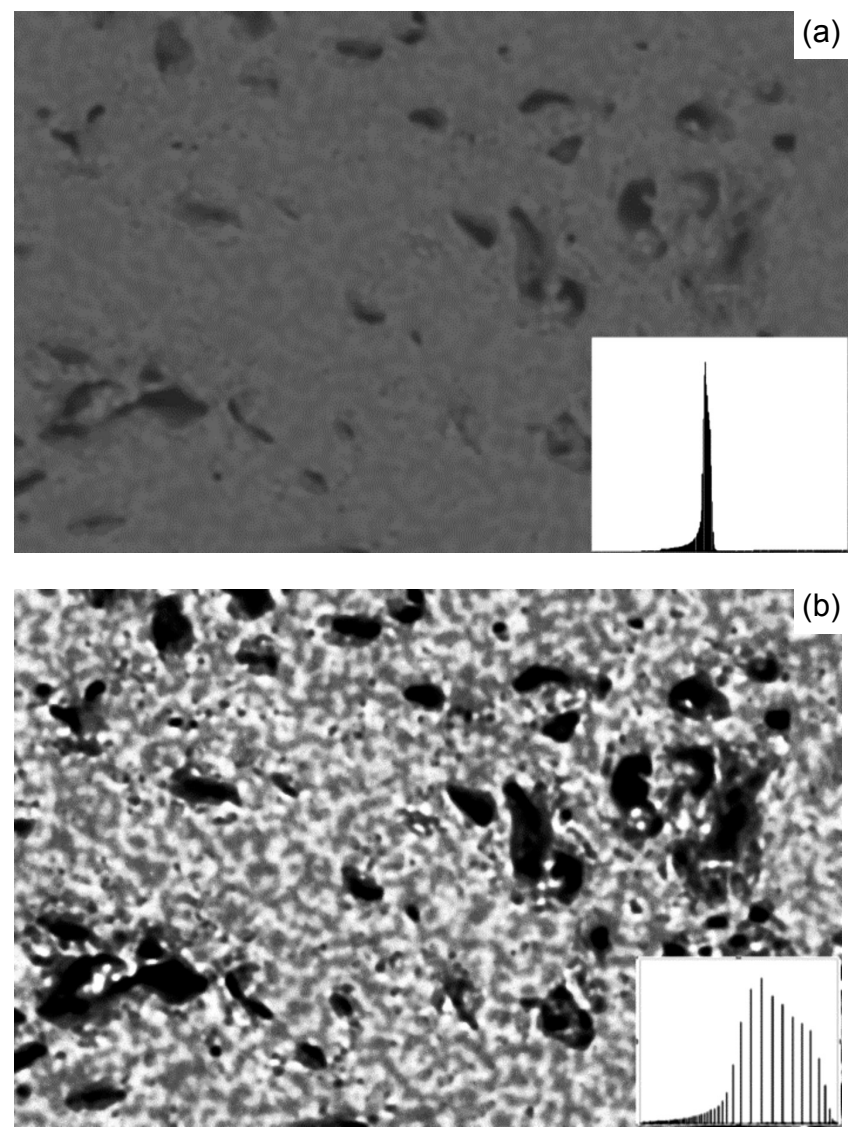

Figura 3: A) Imagem original e seu histograma apresentando apenas um pico; B) Imagem com o contraste melhorado, mostrando 3 regiões distintas representando cada uma das fases.

[Figure 3: A) Original image and its histogram presenting only one peak; B) Image with enhanced contrast, showing 3 different regions indicating each phase.]

comando que transforma em $1 \mathrm{o}$ valor dos pixeis dentro de uma faixa de valores selecionadas e em 0 pixeis que apresentam valores fora desta faixa, foram geradas imagens binárias que representam as redes de NiO, ZEI e Poros, Fig. 4. A partir destas imagens foram determinadas as frações de área ocupada pela fase de interesse na secção analisada, correlacionando com as frações volumétricas

A Tabela I apresenta os resultados de porosidade e fração volumétrica das fases obtidas pela análise de imagem e os resultados obtidos a partir das medidas de porosidade utilizando princípio de Arquimedes, sendo que as frações volumétricas foram calculadas considerando as frações iniciais de cada óxido. Os resultados obtidos a partir da análise de imagem foram bastante próximos aos valores de referência. É importante salientar que a porosidade determinada por análise de imagem considera também os poros fechados, enquanto a medida pelo princípio de Arquimedes considera apenas os poros abertos. Sendo assim, o fato dos valores de porosidade determinados pelas duas 

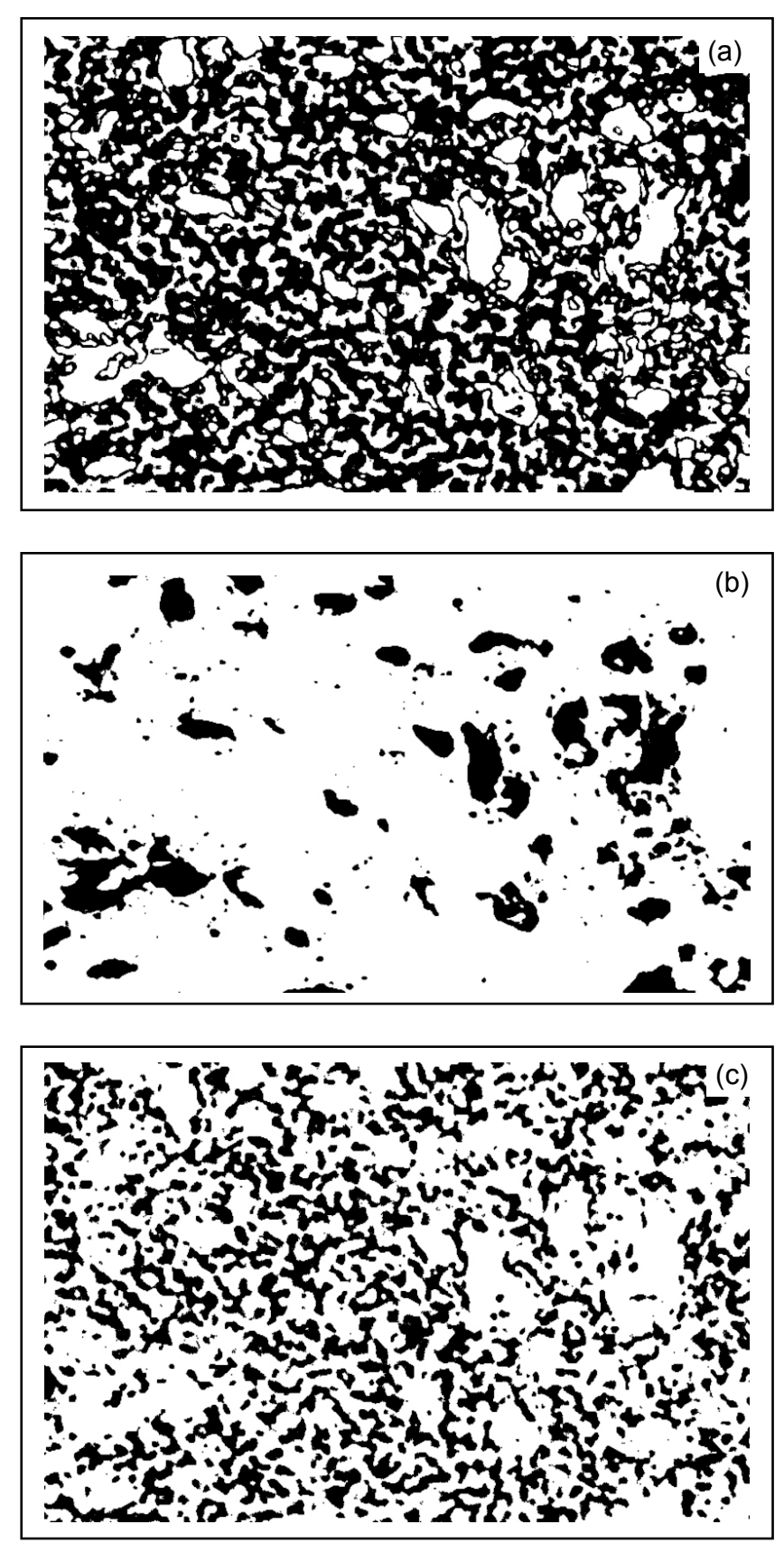

Figura 4: Imagens binárias mostrando as fases: A) NiO, B) Poros e C) ZEI.

[Figure 4: Binary images showing the phases: A) NiO, B) Pores and C) YSZ.]

Tabela I - Resultados das medidas de porosidade e fração volumétrica de fases.

[Table I - Results of porosity and volumetric phase fraction measurements.]

\begin{tabular}{ccc}
\hline Fase & $\begin{array}{c}\text { Análise de Imagem } \\
(\% \mathrm{vol})\end{array}$ & $\begin{array}{c}\text { Princípio de } \\
\text { Arquimedes (\%vol) }\end{array}$ \\
\hline NiO & $50 \pm 7$ & 50,1 \\
ZEI & $32 \pm 2$ & 34,9 \\
Porosidade & $17 \pm 7$ & 15 \\
\hline
\end{tabular}

técnicas serem próximos indica que este compósito poroso possui uma rede de poros interconectados, possibilitando o fluxo de gases quando utilizado como substrato poroso em pilhas a combustível.

A Fig. 5 apresenta uma micrografia de uma pastilha de ZEI sinterizada a $1500{ }^{\circ} \mathrm{C} / 2 \mathrm{~h}$, onde nota-se que existe uma quantidade considerável de poros fechados, que não são quantificados na medida baseada no princípio de Arquimedes.

Aplicando a ferramenta Limiarização é possível evidenciar estes poros fechados, Fig. 6, e quantificá-los. A Tabela II compara os valores de porosidade determinados

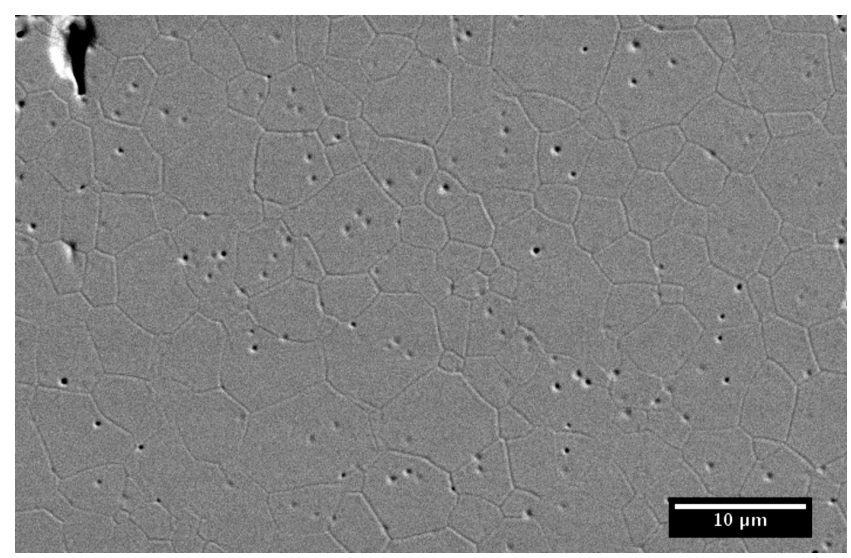

Figura 5: Micrografia obtida em microscópio eletrônico de varredura de uma amostra de ZEI sinterizada a $1500{ }^{\circ} \mathrm{C} / 2 \mathrm{~h}$ e atacada termicamente a $1400{ }^{\circ} \mathrm{C} / 30 \mathrm{~min}$.

[Figure 5: SEM micrograph of a YSZ sample sintered at $1500{ }^{\circ} \mathrm{C} /$ $2 \mathrm{~h}$ and thermally etched at $1400^{\circ} \mathrm{C} / 30 \mathrm{~min}$.]

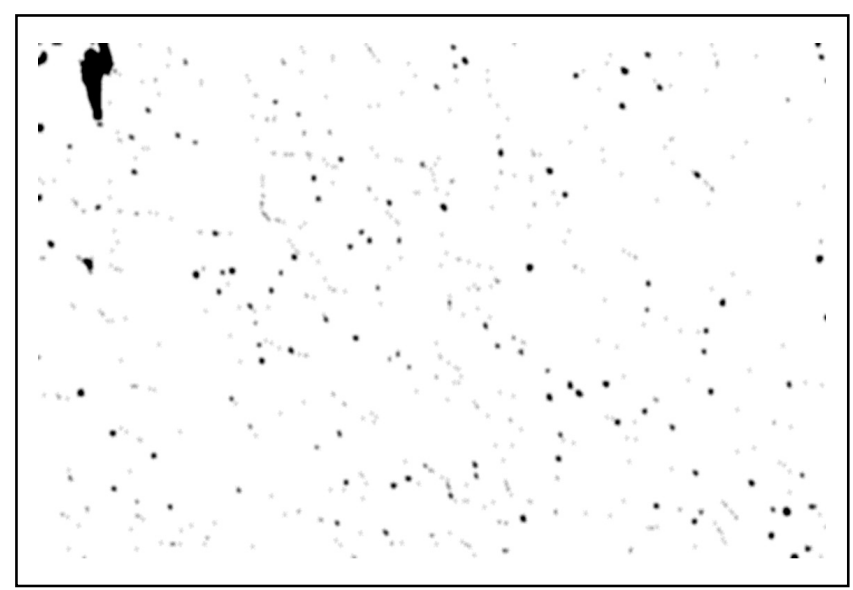

Figura 6: Figura 5 após o comando Limiarização evidenciando os poros.

[Figure 6: Same as Fig. 5 after using Threshold showing the pores.]

Tabela II - Porosidade da pastilha de zircônia sinterizada a $1500^{\circ} \mathrm{C} / 2 \mathrm{~h}$.

[Table II - Porosity of a zirconia pellet sintered at $1500^{\circ} \mathrm{C} / 2 \mathrm{~h}$.]

\begin{tabular}{cc}
\hline Princípio de Arquimedes & Análise de Imagem \\
\hline $0,17 \pm 0,01 \%$ & $1,5 \pm 0,2 \%$ \\
\hline
\end{tabular}


por análise de imagem com o obtido utilizando o princípio de Arquimedes. A diferença entre os valores é devida ao fato de que a técnica baseada no princípio de Arquimedes mede somente os poros abertos, enquanto a análise de imagem considera também os poros fechados.

\section{Quantificação do tamanho de grãos}

A análise do tamanho de grãos utilizando o programa ImageJ foi realizada em micrografia obtida por microscopia eletrônica de varredura de ZEI sinterizada a $1500{ }^{\circ} \mathrm{C} / 2 \mathrm{~h}$, Fig. 5. Observa-se na imagem que os contornos de grãos não estão bem definidos além de apresentar ruídos e diferenças de brilho que podem interferir na análise. Neste caso se faz necessária a utilização de filtros para eliminar estes defeitos e evidenciar os contornos de grão para que a análise possa ser realizada com maior precisão.

Para corrigir as diferenças de iluminação utilizou-se o comando "Subtract Background" (Subtrair Fundo). Este comando ébaseado no algoritmo "Rolling Ball" desenvolvido por S. Sternberg e consiste em gerar uma imagem de fundo a partir da média de valores de aproximadamente 50 pixeis de raio a partir de um pixel central [16]. Esta imagem de fundo é subtraída da imagem original, corrigindo diferenças de iluminação, Fig. 7.

Após a correção de iluminação, utilizou-se o comando

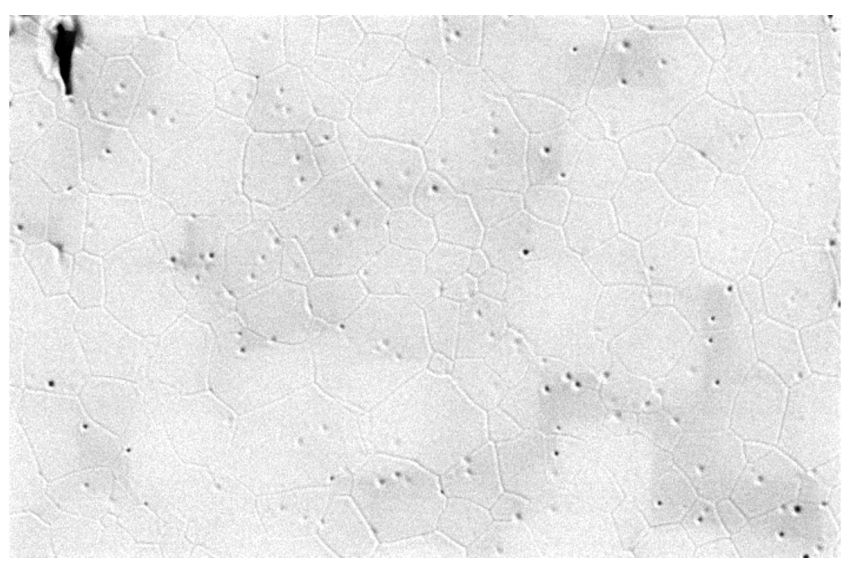

Figura 7: Figura 5 após a utilização do filtro Subtrair Fundo (Subtract Background).

[Figure 7: Figure 5 after using Subtract Background filter.]

"Find Edges" (Encontre Bordas), que atua indicando gradientes de variação de cor, acentuando assim as interfaces [17]. A Fig. 8 mostra a imagem resultante contendo os contornos de grão em evidência. Esta imagem ainda contém ruídos e os contornos de grãos estão "abertos", sendo contados apenas como um objeto pelo programa.

Utilizando o comando "Make Binary" (Transformar em Binária), a imagem foi binarizada e a partir dos comandos com imagem binária obteve-se a Fig. 9, que representa os contornos de grãos da amostra. Os comandos binários utilizados foram "Erode", que remove pixeis das bordas de objetos brancos; "Dilate", que adiciona pixeis às bordas de

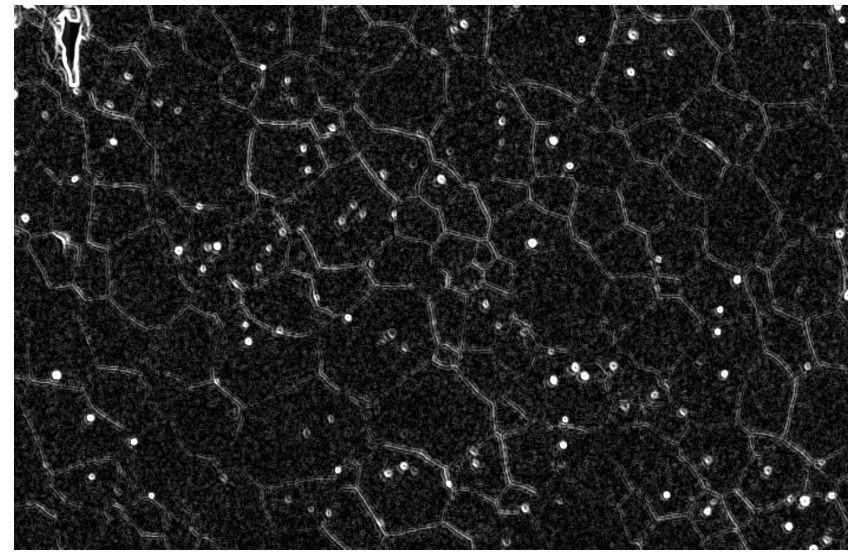

Figura 8: Figura 7 após a aplicação do filtro Encontre Bordas (Find Edges).

[Figure 8: Figure 7 after the application of Find Edges filter.]

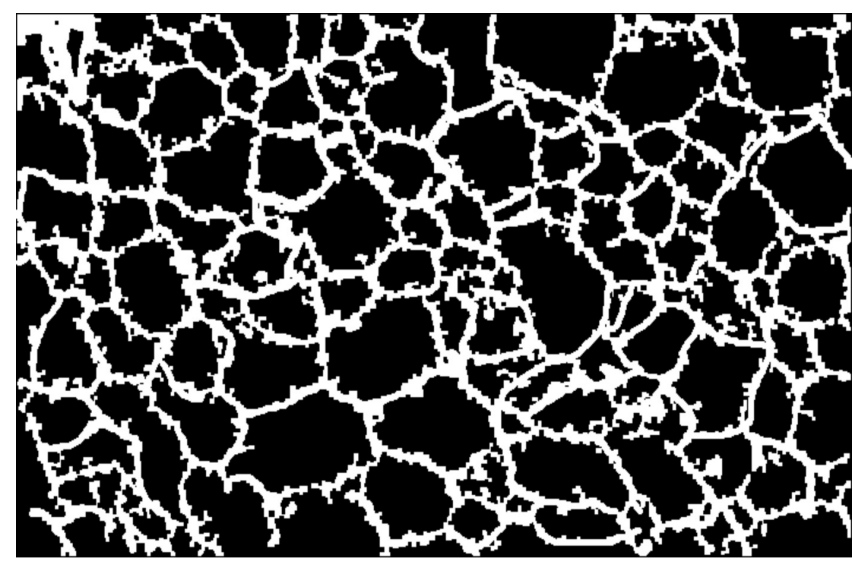

Figura 9: Imagem binária enfatizando os contornos de grãos. [Figure 9: Binary image emphasizing the grain boundaries.]

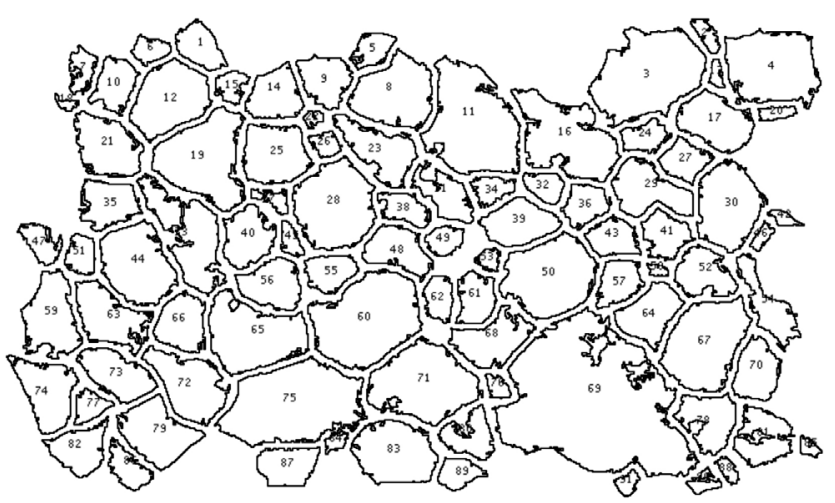

Figura 10: Objetos contados na medida de tamanho de grãos. [Figure 10: Counted objects in the grain size measurement.]

objetos brancos e "Fill Holes" que preenche pixeis brancos contidos em um fundo preto [18]. A partir da imagem binária, Fig. 9, fez-se a medida de diâmetro de Feret, que indica a distância entre duas retas paralelas que tangenciam o objeto, excluindo os grãos dispostos na borda da imagem. A Fig. 10 mostra os grãos que foram considerados na análise.

Aplicando o procedimento descrito acima foi obtido 4,6 
$\pm 0,2 \mu \mathrm{m}$ para o tamanho médio de grão, assim como as curvas de distribuição de tamanho de grãos e distribuição acumulada de tamanho de grãos, Fig. 11.

Distribuição acumulada de tamanho de grãos
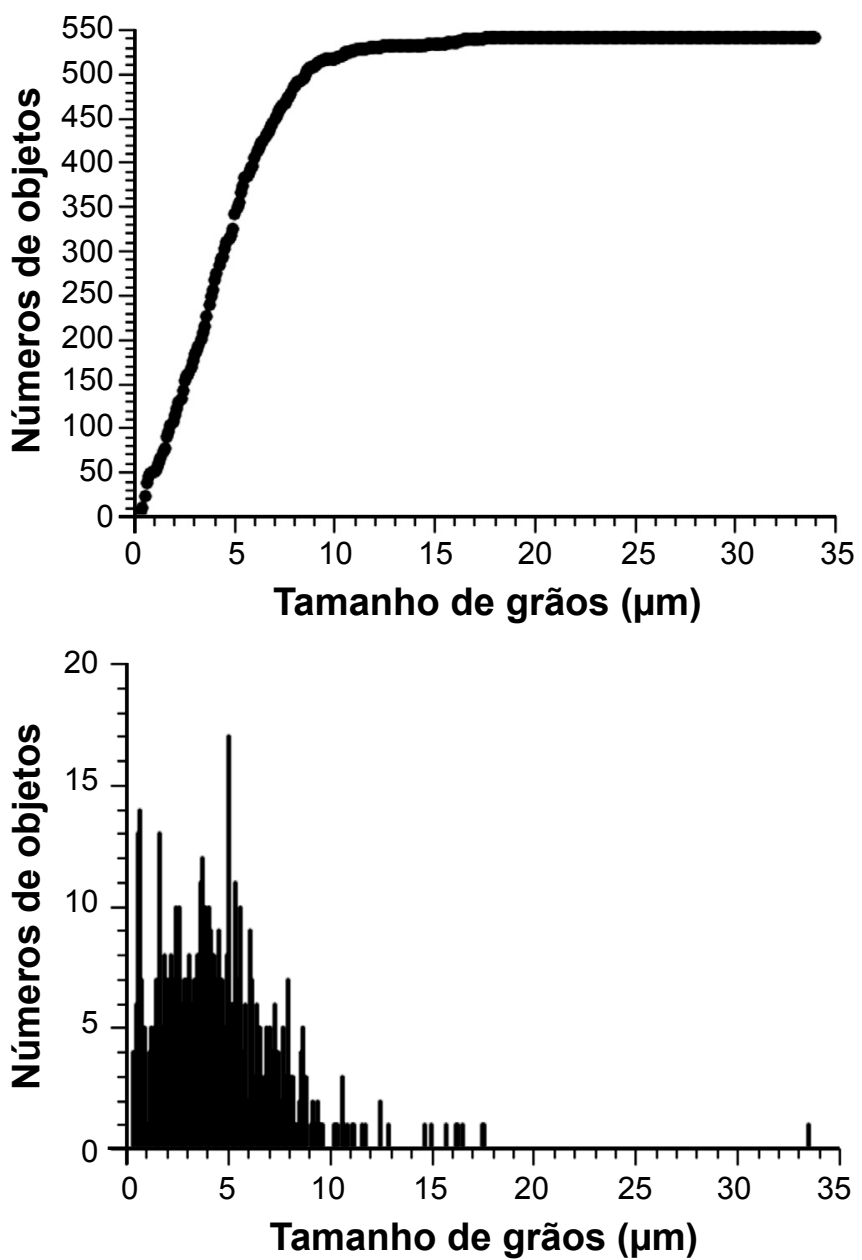

Figura 11: A) Distribuição de tamanho de grãos. B) Distribuição acumulada de tamanho de grãos.

[Figure 11: A) Grain size distribution. B) Accumulated grain sized distribution.]

\section{CONCLUSÕES}

A análise de imagem automatizada gera resultados com grande rapidez e precisão sem interferência do operador. Utilizando filtros que alteram os valores dos pixeis podem-se evidenciar regiões de interesse, de maneira que o programa possa diferenciar os objetos e realizar a análise. A medida de porosidade considera, além dos poros abertos, os poros fechados. Em relação ao tamanho de grãos, a partir desta técnica pode-se determinar não só seu tamanho médio, como também sua distribuição.

\section{AGRADECIMENTOS}

Os autores agradecem ao CNPq e à FINEP pelo apoio financeiro e ao Laboratório de Caracterização Estrutural da UFSCar (LCE/UFSCar) pela disponibilização de sua infraestrutura.

\section{REFERÊNCIAS}

[1] S. Amelinckx, D. Van Dyck, J. van Landuyt, G. van Tendeloo, Handbook of Microscopy - Applications in Materials Science, Solid-state Physics and Chemistry, VCH Verlagsgesellschaft GmbH, Weinheim, Alemanha (1997) 1. [2] ImageJ - Introduction, disponível em: <http://rsbweb. nih.gov/ij/docs/intro.html>, acessado em 21/03/2010.

[3] L. Wojnar, Image Analysis: Application in Materials Engineering, CRC Press, Boca Raton, FL, EUA (1999) 1.

[4] Z. Latala, L. Wojnar, Mater. Charact. 46 (2001) 227233.

[5] D. Simwonis, F. Tietz, D. Stover, Solid State Ionics 132 (2000) 241-251.

[6] R. Krashanitsa, H. Luo, W. Chen, S. Shkarayev, Int. J. Damage Mech. (2009) 1-15.

[7] J.-H. Lee, H. Moon, H. -W. Lee, J. Kim, J. -D. Kim, K. -H. Yoon, Solid State Ionics 148 (2002) 15-26.

[8] K. -R. Lee, S. H. Choi, J. Kim, H. -W. Lee, J. -H. Lee, J. Power Sources 140 (2005) 226-234.

[9] K. -R. Lee, Y. S. Pyo, B. S. So, S. M. Kim, B. K. Lee, J. H. Hwang, J. Kim, J. -H. Lee, H. -W. Lee, J. Power Sources 158 (2006) 45-51.

[10] Z. Shan, A. M. Gockale, Int. J. Plasticity 20 (2004) 1347- 1370 .

[11] J. G. Berryman, S. C. Blair, J. Appl. Phys. 60, 6 (1986) 1930- 1938.

[12] ASTM Standard E 562, 2002: Standard Test Method for Determining Volume Fraction by Systematic Manual Point Count, ASTM International, West Conshohocken, PA, EUA (2002) DOI: 10.1520/ E0562-02, www.astm.org. [13] ASTM Standard E 1382, 1997: Standard Test Methods for Determining Average Grain Size Using Semiautomatic and Automatic Image Analysis, ASTM International, West Conshohocken, PA, EUA (2002) DOI: 10.1520/E1382-97, www.astm.org.

[14] Sigma Filter, disponível em <http://rsb.info.nih.gov/ ij/plugins/sigma-filter.html $>$, acessado em 29/04/2009.

[15] Enhance Contrast- ImageJ Documentation, disponível em <http://imagejdocu.tudor.lu/doku.php?id=gui:process: enhance contrast $>$, acessado em 17/11/2009.

[16] Subtract Background- ImageJ Documentation, disponível em $<$ http://imagejdocu.tudor.lu/doku php?id=gui: process:subtract_background $>$ acessado em 17/11/2009.

[17] Sobel operator - Wikipedia, disponível em $<$ http:// en.wikipedia.org/wiki/Sobel_operator $>$ acessado em 17/11/2009.

[18] Binary - ImageJ Documentation, disponível em $<$ http:// imagejdocu.tudor.lu/doku.php?id=gui:process:binary> acessado em 17/11/2009

(Rec. 08/02/2010, Rev. 19/04/2010, Ac. 22/05/2010) 